\title{
Effect of Threonine and the Bioactive Com- ponent of Saccharomyces Cerevisiae on the Productive Performance of the Broiler Cobb 500
}

\section{Efectos de Treonina y Componentes Bioac- tivos de Saccharomyces cerevisiae Sobre la Producción de Aves Cobb 500}

I International Seminar of Livestock and Agroindustrial Production ESPOCH 2020

Corresponding Author:

P. Toalombo Vargas

ptoalombo@espoch.edu.ec

Published: 2 September 2021

Production and Hosting by

Knowledge E

(c) J. Encalada Álvarez et al. This article is distributed under the terms of the Creative Commons Attribution License, which permits unrestricted use and redistribution provided that the original author and source are credited.

\author{
J. Encalada Álvarez ${ }^{1}$, P. Toalombo Vargas², C. Vimos Abarca², J. Trujillo \\ Villacís ${ }^{2}$, and L. Silva Deley ${ }^{3}$ \\ ${ }^{1}$ Investigadora independiente \\ ${ }^{2}$ Carrera de Zootecnia, Facultad de Ciencias Pecuarias, Escuela Superior de Chimborazo, \\ Riobamba, Ecuador \\ ${ }^{3}$ Medicina Veterinaria, Universidad Técnica de Cotopaxi, Latacunga, Ecuador
}

\section{Abstract}

This study was conducted in Chimborazo province, Riobamba Canton to evaluate the effect of threonine and the bioactive component of Saccharomyces cerevisiae on the productive performance of the broiler Cobb 500. A total of 270 one-day-old broiler chicken of both sexes were included, which corresponded to an experimental unit size of 15 birds. Two growth promoters were used for the treatments - T1: Threonine (aminoacid) $200 \mathrm{~g} / \mathrm{Tn}$; and T2: bioactive oligosaccharides, obtained from the cell wall of selected strains of S. Cerevisiae (probiotic) 750 $\mathrm{g} / \mathrm{Tn}$. These were compared to a control group. The data were analyzed through Analysis of Variance (ADEVA). The separation of means was performed using the Tukey statistic at a level of significance of $p<0.05$ and $p<0.01$. The data were processed using the Infostat software version 2010. The results showed that the best productive yields were with treatment 2; the values for this treatment were: weight at 28 days: $1369.42 \mathrm{~g}$; weight gain at 28 days: $48.90 \mathrm{~g}$; food conversion at 28 days: 1.39 points; carcass weight: $2527.05 \mathrm{~g}$; and yield to the carcass: 83.85\%. Through the economic analysis, it was determined that the highest cost-benefit index was 1.30 USD with the application of T2. So according to the results, a better use of the nutrients that are present in the feed is achieved when bioactive components of $S$. cerevisiae are supplied in the diet of broiler chickens.

Keywords: Threonine, Saccharomyces cerevisiae, productive performance, broiler, Cobb 500.

\section{Resumen}

Se realizó un experimento en la provincia de Chimborazo, Cantón Riobamba, para evaluar los efectos de treonina y componentes bioactivos de Saccharomyces cerevisiae sobre el comportamiento productivo en aves Cobb 500. Se utilizaron 270 pollitos mixtos Cobb 500 de un día de edad de ambos sexos, con un tamaño de unidad experimental de 15 aves. Para los tratamientos se manejaron dos promotores de crecimiento, T1: Treonina (aminoácido) 200 g/Tn; y T2: Oligosacáridos bioactivos, obtenidos a partir de la pared celular de cepas seleccionadas de S. Cerevisiae (probiótico) $750 \mathrm{~g} / \mathrm{Tn}$; frente a un testigo (TO). Los datos obtenidos fueron sometidos a Análisis de Varianza (ADEVA); la separación de medias se realizó mediante el 
estadístico Tukey a un nivel de significancia $(p<0,05)$ y $(p<0,01)$; los datos se procesaron mediante el software Infostat versión 2010. Los resultados muestran los mejores rendimientos productivos con el Tratamiento 2, para los parámetros: peso a los 28 días 1369,42 g; ganancia de peso a los 28 días 48,90 g; y conversión alimenticia a los 28 días con 1,39 puntos; así como peso a la canal $2527,05 \mathrm{~g}$; y rendimiento a la canal $83,85 \%$. Mediante el análisis económico se determinó que el mayor índice beneficio costo fue de 1,30 USD con la aplicación del T2. Lo que brinda un indicativo que mediante el suministro de componentes bioactivos de $S$. cerevisiae en la dieta de pollos broiler, se logra un mejor aprovechamiento de los nutrientes que se encuentran presentes en el alimento, lo que se refleja en los parámetros productivos.

Palabras Clave: treonina, Saccharomyces cerevisiae, comportamiento productivo, broilers, Cobb 500

\section{Introducción}

En las diferentes explotaciones de producción avícola es habitual el uso de aditivos como promotores de crecimiento, ya que ayudan a incrementar la eficacia de los nutrientes que se encuentran en las dietas alimenticias, como también promueve el crecimiento y una mayor productividad de los animales, entre los productos utilizados como aditivos [1] tenemos los probióticos, aminoácidos, así como también prebióticos, enzimas, vitaminas y sales minerales [2, 3].

El uso continuo de antibióticos como promotores de crecimiento (APC) en la producción avícola, se ha asociado con un aumento en la aparición de bacterias resistentes a dichos fármacos, causando efectos negativos sobre la salud y el bienestar tanto de los animales como de los consumidores, así como en la sustentabilidad y cuidado del medio ambiente [4]; es por esto que se ha prohibido el uso de estas sustancias en la dieta animal en varios países europeos, [5] y el cual tomó relevancia, ya que se generó mecanismos de resistencia al acumularse sus residuos en carne y huevos [2]. El uso de APC antibiótico disminuye la presencia de IgA secretoria, esto se asocia a la acción antibiótica del BMD (bacitracina metileno disalicilato), ya que éste afecta a la microbiota normal de las aves y como consecuencia podría estar afectando la respuesta inmune de la mucosa intestinal. Mientras que en estudios realizados donde se suministró probióticos, las aves presentaron incrementos en los valores relativos de IgA, lo que corresponde con el proceso de maduración de la respuesta inmune [5]. Por lo que se ha visto la necesidad de buscar alternativas con el fin de poder sustituir de forma eficiente y económica a los APC, pero que a su vez representen el mismo impacto sobre los parámetros productivos y de salud intestinal en los pollos de engorde [3].

La alimentación en la crianza de aves de carne, es una tarea importante, ya que el costo representa más de dos tercios del costo total de la producción avícola [6]. Por lo tanto, incrementar la eficiencia alimenticia (es decir, convertir la masa de alimento en masa corporal de ave) resulta significativo para la rentabilidad de la granja [7]. Por lo que para optimizar la conversión alimenticia, es decir, mejorar la utilización metabólica de los nutrientes de la dieta del ave, se basa en gran medida a un intestino o tracto gastrointestinal (TGI) saludable, ya que un intestino sano puede resultar en una mejor 
digestión del alimento, absorción de nutrientes a través de sus membranas epiteliales $[8,9]$.

El tracto digestivo gastrointestinal (TGI), es un tubo que se encuentra recubierto por células epiteliales especializadas, las cuales se extienden continuamente, y que a su vez recubren la piel. A lo largo del TGI, las células se van diferenciando para ejercer funciones especiales que incluyen la trituración física del alimento, secreción de fluídos, electrolítos, mucusa, enzimas; así como también digestión, absorción, transporte de nutrientes. La principal función es la de defensa, mediante la mucosa intestinal que forma gran cantidad de pliegues microscópicos en forma de dedos, a los cuales se denomina 'vellosidades intestinales', y que a su vez éstos presentan micro vellosidades. Al ser el sistema digestivo abierto, este se encuentra en permanente contacto con el exterior y los patógenos, por lo que está sujeto a permanentes agresiones; el alimento suministrado a los animales está en constante relación con el intestino, así como también con las secreciones, fluidos y enzimas que este genera, formando el denominado 'quimo', si a ello se suma los microorganismos propios o ingeridos, los cuales pueden ser infeccioso o no; razón por la cual se debe brindar una protección extra al TGI, para que pueda de tal manera absorber y aprovechar los nutrientes [10].

El segmento proximal del tracto digestivo de las aves muestran una mejor eficiencia digestiva, ya que ventrículos y proventrículos se encuentran más desarrollados; todo lo contrario, a la parte distal del tracto digestivo que contienen segmentos intestinales más cortos (duodeno, íleon, yeyuno); por lo que los animales muestran un tiempo de retención de alimento mucho más largo en su proventrículo, ventrículo y ciego, lo que aumenta el tiempo de molienda, mezcla con enzimas digestivas y absorción de nutrientes. Como resultado de estas diferencias, las aves eficientes excretan una mayor proporción de partículas finas en sus heces [11].

Los probióticos, prebióticos y aminoácidos actúan a nivel de la microbiota intestinal de las aves, la misma que está compuesta por una mayoría de microorganismos anaeróbicos que trabajan en simbiosis con su huésped, realizando funciones esenciales que contribuyen a la regulación de toda la fisiología y metabolismo, para la expresión de caracteres de interés productivo [12-14]. Siendo entonces la salud intestinal, el resultado de interacciones funcionales complejas entre los microbios intestinales y la inmunidad del huésped [15].

Las levaduras han sido incorporadas en las dietas animales como aditivos ya que actúan como promotores de crecimiento probióticos, teniendo una fuente rica en proteínas de alto valor biológico [16] por estar formadas de aminoácidos esenciales y una excelente fuente de vitaminas del complejo B [17]. En la actualidad el estudio de la síntesis, composición y ensamblaje de la pared celular de Saccharomyces cerevisiae, ha ido en aumento; la misma se encuentra constituida principalmente por polisacáridos en proporción de $58 \%$ en $\beta$-glucanos, $40 \%$ en manoproteínas y un $2 \%$ de quitina [18]. Varios métodos analíticos se han perfeccionado para cuantificar los polisacáridos presentes en la pared celular de las levaduras, con el propósito de observar el contenido y variación de estos compuestos, en diferentes cepas de levaduras nativas y modificadas, relacionando la variación de estos polisacáridos con la resistencia de la célula a la ruptura mecánica o enzimática y a la acción de los antibióticos [19]. Su uso sobre las 
variables de comportamiento productivo, calidad y rendimiento a la canal en diferentes estirpes de aves destinadas para producción de carne, han sido evaluadas en distintas presentaciones, ya sea sola y/o combinada con otros probióticos, prebióticos, enzimas, antibióticos promotores de crecimiento; así como su reemplazo por parte del núcleo vitamínico-mineral. Dando como resultado mejora en los parámetros productivos de pollos broiler, incluso cuando es reemplazado hasta un tercio del núcleo vitamínico mineral del alimento balanceado; o en combinación de otros aditivos ya sean APC o probióticos, prebióticos. Los efectos positivos pueden deberse a su composición, básicamente a los mananooligosacáridos de su pared celular, que actuarían como biorreguladores de la flora intestinal del ave, y por ende puede presentar acción curativa ó preventiva.

Las dietas ricas en fibra influyen en la mucosa del intestino delgado, por lo que las fracciones insolubles favorecen los efectos abrasivos sobre la barrera mucosa intestinal [20]. La mucosa intestinal es una capa dinámica, compuesta predominantemente de mucina, que protege a las células epiteliales del daño enzimático y la invasión bacteriana [21]. Se ha demostrado que la fibra dietética sufre cambios en la cantidad y tipo de mucina [22]. La estructura de la mucina está compuesta por proteínas y algunos aminoácidos como la serina, treonina (Thr) y cisteína. El papel destacado de Thr en la producción de mucina se debe a que Thr constituye hasta el 11\% de los aminoácidos de la mucina. Como tal, la dinámica de la mucina en el intestino podría ser sensible a la disponibilidad de Thr. La Treonina es el tercer aminoácido más limitado en pollos de engorde alimentados con dietas a base de maíz, particularmente en dietas bajas en proteína cruda [23]; Este aminoácido participa en procesos metabólicos importantes, como la formación de ácido úrico y la síntesis de proteínas. Sin embargo, las aves de corral no sintetizan suficiente Thr, lo que hace que este aminoácido sea nutricionalmente necesario para ser utilizado en la dieta de los pollos de engorde; además juega un papel fundamental en el mantenimiento de la integridad de la barrera intestinal y la síntesis de mucina [24, 25]. Se encuentra en altas concentraciones como pérdidas de proteínas endógenas, particularmente en las mucinas en las que la treonina representa el $16 \%$ del total de aminoácidos [23]. Este aminoácido es un componente importante de la $\gamma$-globulina plasmática en aves, cerdos, conejos y humanos, y es crucial para la función del sistema inmunológico [26] puede promover el crecimiento de órganos inmunes, estimular la síntesis de inmunoglobulinas, incluidas inmunoglobulina A ( $\lg A)$, inmunoglobulina $G(\lg G)$ e inmunoglobulina A secretora (SIgA), por ende mejora la respuesta inmunitaria y alivia el estrés inmunológico inducido por exposición a microorganismos [27-30]. La evaluación de la inmunidad de los pollos de engorde mediante la evaluación de anticuerpos es razonable, debido al hecho de que la disminución de los anticuerpos humorales podría aumentar la susceptibilidad de los pollos a las enfermedades [22], Wils-Plotz et al. [31] declararon que la inclusión dietética de $70 \mathrm{~g} / \mathrm{kg}$ de fibra purificada (celulosa o pectina) no altera el requisito de Thr en pollos de engorde. Además, se informó que Thr influye en la respuesta inmune intestinal en pollos de engorde infectados con Eimeria máxima.

Es por ello que, en los últimos años, se ha incrementado la búsqueda de agentes naturales que puedan actuar como promotores de crecimiento, o bien que permitan el 
control de algunos microorganismos patógenos. Por lo que en la presente investigación se utilizó treonina (aminoácido) y componentes bioactivos de S. cerevisiae (prebiótico), con la finalidad de incrementar inmunidad, flora intestinal y mejorar los parámetros productivos en pollos broiler de la línea Cobb 500.

\section{Materiales y Métodos}

\subsection{Localización y duración del experimento}

La presente investigación se llevó a cabo en la provincia de Chimborazo, Cantón Riobamba, comunidad Socorro Bajo, ubicada a una Altitud de 1220 m.s.m.n., temperatura $16^{\circ} \mathrm{C}$; Precipitación $470 \mathrm{~mm} / \mathrm{mes}$; Humedad relativa $60 \%$, información tomada de la Estación Agrometereológica de la Facultad de Recursos Naturales ESPOCH -2016. el tiempo de duración del proyecto fue de 60 días.

\subsection{Tratamientos y diseño experimental}

Se utilizaron 270 pollitos mixtos (macho y hembra) de un día de edad de la estirpe Cobb 500, que se distribuyeron bajo un diseño completamente al azar en tres tratamientos, con seis repeticiones y un tamaño de unidad experimental de 15 aves. Para los tratamientos se manejaron dos promotores de crecimiento: T1: Treonina (aminoácido); y T2: Oligosacáridos bioactivos, obtenidos a partir de la pared celular de cepas seleccionadas de S. Cerevisiae (prebiótico), frente a un testigo (TO). En las siguientes dosis: T1: $200 \mathrm{~g} / \mathrm{Tn}$; T2: $750 \mathrm{~g} / \mathrm{Tn}$ versus TO: Testigo.

Se aplicó un sistema de crianza semi-intensivo, basados en el Manual de Manejo de la Línea Cobb 500, (MANUAL) Se adquirió los pollitos de 1 día de edad, para determinar un peso promedio inicial. Se colocó a los pollitos en un círculo de crianza que debe estar preparado mediante una desinfección, colocación de cama unos 10 días antes de la llegada. Para brindarles un ambiente ideal a los individuos, se tuvo un adecuado sistema de ventilación por cortinas, iluminación mediante focos y una confortable calefacción con el uso de criadora que mantendrá una temperatura de $32^{\circ} \mathrm{C}$. A partir del primer día se empezó el empleo de los diferentes promotores de crecimiento. Cada uno de los tratamientos recibió diferentes promotores de crecimiento en el alimento, para ello se elaboró tres tipos de balanceados para cada etapa; divida en: Inicial desde el primer día hasta los 14 días de edad; concentrado de crecimiento de 15 a 28 días de edad; y concentrado finalizador desde los 29 hasta los 49 días de edad. Así también se distribuyeron los comederos y bebederos, en función del número de aves para suministrar el balanceado y el agua de bebida que fue a voluntad; en el agua de bebida se colocó los antibióticos y vitaminas para la llegada de los pollitos. El manejo sanitario se realizó mediante el siguiente programa de vacunación dentro de la granja: Día 7 = Bronquitis y Newcastle - Vía ocular; Día 14 = Gumboro - Vía Agua; Día $21=$ Bronquitis y Newcastle - Vía ocular. 


\subsection{Análisis estadísticos y pruebas de significancia}

Los datos numéricos de campo generados en la propuesta investigativa fueron sometidos a Análisis de Varianza (ADEVA); la separación de medias se realizó mediante el estadístico Tukey a un nivel de significancia $(p<0,05)$ y $(p<0,01)$; los datos se procesaron mediante el software estadístico Infostat versión 2010.

\section{Resultados y Discusiones}

\subsection{Peso inicial, $g$}

Los pesos iniciales de las aves para cada tratamiento fueron homogéneos, con medias de 48,83; 49,42 y 49,83 g para T0, T1 y T2, en su orden. Tabla 1 Datos similares reporta [32] en la misma línea genética de aves al día 1 de edad, ya que dicho peso se encuentra determinado en el Manual de Manejo Cobb 500.

\subsection{Peso a los 14 días, $g$}

El peso a los 14 días (fase inicial), no presentaron diferencias estadísticas significativas ( $p$ $>0,05)$, con respecto a los promotores de crecimiento utilizados; con pesos promedios de T0: 299,08 g; T1:304,08 g; T2: 308,58 g (Tabla 1). Valores análogos, que a su vez no presentan diferencias significativas, menciona [5] en su estudio comparativo realizado entre promotores de crecimiento antibiótico y una base de un extracto vegetal polifenólico en la misma línea genética de aves. Upendra y Yathiraj [33] observó que al aplicar S. Cerevisiae a la dieta, el peso vivo del ave mejoró, pero sin diferencias estadísticas, lo que sugiere que no existe una sinergia positiva de estos dos promotores de crecimiento en la etapa inicial. En relación a la adición de treonina se determinaron valores superiores a los de la presente investigación debido al incremento de treonina en la dieta $(1 \mathrm{~g} / \mathrm{kg}$ ) en una línea comercial diferente (Arbor Acres Plus) [23].

\subsection{Peso a los 28 días, $\mathbf{g}$}

Para el peso a los 28 días se obtuvieron diferencias estadísticas altamente significativas $(p<0,01)$, entre los promotores de crecimiento utilizados; siendo el mayor T2: 1369,42 g; seguido por T1:1324,08 g y T0:1316,58 g. Lo que demuestra que los componentes bioactivos de $S$. Cerevisiae mejora el peso en la etapa de crecimiento considerablemente, así también afirma [34], donde menciona que el uso de dicho prebiótico representa una nueva alternativa tecnológica para mejorar la eficacia alimentaria, esto puede ser explicado por un mayor aprovechamiento de nutrientes, que pasan por el yeyuno, cuya altura de las vellosidades intestinales se ven incrementadas a través del aumento del contenido de RNA de los enterocitos; el alimento es fermentado por la microflora endógena, debido a que son ingredientes que benefician el hospedero por estimulación selectiva de crecimiento y actividad bacteriana, además existe una disminución de bacterias entero patógenas que impidan aumentar el dominio de la 


\section{Table 1}

Efectos de treonina y componentes bioactivos de Saccharomyces cerevisiae sobre la producción de aves Cobb 500.

\begin{tabular}{|c|c|c|c|c|c|c|c|c|c|}
\hline \multirow[t]{2}{*}{ Variable } & \multicolumn{6}{|c|}{ Tratamientos } & \multirow[t]{2}{*}{ E.E } & \multirow[t]{2}{*}{ Prob. } & \multirow[b]{2}{*}{ Sig. } \\
\hline & TO & & T1 & & T2 & & & & \\
\hline Peso inicial, g & 48,83 & & 49,42 & & 49,83 & & & & \\
\hline Peso final, 14 días & 299,08 & a & 304,08 & a & 308,58 & a & 5,5963 & 0,5005 & ns \\
\hline Peso final, 28 días & 1316,58 & $b$ & 1324,08 & $b$ & 1369,42 & a & 7,2855 & 0,0002 & $* *$ \\
\hline Peso final, 49 días & 2985,25 & a & 2992,17 & a & 3013,75 & a & 18,3223 & 0,5304 & ns \\
\hline $\begin{array}{l}\text { Ganancia de } \\
\text { peso, } 28 \text { días }\end{array}$ & 47,02 & $b$ & 47,28 & $b$ & 48,90 & a & 6,2855 & 0,0003 & $* *$ \\
\hline $\begin{array}{l}\text { Conversión } \\
\text { alimenticia, } 28 \\
\text { días }\end{array}$ & 1,44 & $b$ & 1,43 & $b$ & 1,39 & a & 5,3743 & 0,0002 & $* *$ \\
\hline
\end{tabular}

${ }^{* *}$ Altamente significativo; E.E.: Error Estándar; ns: No significativo.

Prob. > 0,05: No existen diferencias estadísticas; Prob. < 0,05: Existen diferencias estadísticas; Prob. < 0,01: Existen diferencias altamente significativas.

Medias con letras iguales en una misma fila no difieren estadísticamente de acuerdo a la prueba de Tukey.

flora bacteriana benéfica, siendo una característica propia del modo de actuar de los componentes activos (mánanos y glucanos), que se encuentran presentes en las paredes celulares de S. Cerevisiae [35], que propician una mayor actividad de las enzimas maltasa, leucina aminopeptidasa y fosfatasa alcalina en el yeyuno, mejorando la descomposición azúcares y almidones, aliviando el trabajo excesivo del páncreas.

En cuanto a la inclusión de treonina; Saadatmand et al. [36] reporta efectos similares tomando en consideración que se experimentó en broiler Ross 308, pero en un microclima controlado para ambos sexos; mientras que resultados superiores se observaron en la investigación obtenida por [37] debido a que el estudio se realizó en machos de la línea Ross 308, siendo importante mencionar que por dimorfismo sexual los machos presentan una mayor eficiencia productiva; a su vez el clima y temperatura (cálido húmedo) difieren en cuanto a la presente investigación.

Cabe mencionar que alrededor de las tres cuartas partes de todas las células inmunológicas en el cuerpo del animal están localizadas dentro del intestino como parte del tejido linfoide; proporcionando protección inmunológica, tanto específica como no específica, de manera de proteger la superficie del tracto gastrointestinal [38].

Las diferencias significativas alcanzadas en el presente trabajo, también pueden deberse a lo demostrado en experimentos previos donde se utilizaron productos prebióticos altamente purificados ( $\beta$ 1-3 y $\beta$ 1-6-glucano de $S$. cerevisiae, en líneas comerciales como Ross, Hubbard, HE21EB34, además de la influencia de las condiciones de alojamiento para las aves (ventilación forzada con control de temperatura, iluminación artificial con control del tiempo de exposición) a diferencia de nuestro experimento, que se realizó en condiciones semi-industriales [39]. 


\subsection{Peso a los 49 días}

El peso a los 49 días de los pollos broiler, no presentaron diferencias estadísticas significativas $(p>0,05)$, entre los tratamientos, los promedios por cada tratamiento fueron; T2 3013,75 g; T1: 2992,17 g; T0: 2985,25 g, varios autores indicaron resultados similares; López [40] estudió la inclusión de promotores de crecimiento antibiótico versus S. Cerevisiae, pared celular y extractos; posiblemente esto se deba a que la levadura natural permite un control y establecimiento de una microflora beneficiosa en los animales y una disminución paulatina de la potencialmente enteropatógena. Es importante mencionar que los pesos obtenidos en el experimento realizado, son superiores a lo que obtuvo [41] mismo que utilizó pared celular de S. Cerevisiae a una menor altura.

\subsection{Ganancia de peso de los pollos broiler a los 28 días, g}

La variable ganancia de peso a los 28 días de evaluación, presentaron diferencias estadísticas altamente significativas $(p<0,01)$, llegan a mostrarse el tratamiento más eficiente con la aplicación del T2: 48,90; al ser contrastado con T1: 47,28 y T0:47,02 (Tabla 1); a lo que se puede indicar que el uso de componentes bioactivos de la pared celular S. Cerevisiae corrobora en la mitigación de bacterias patógenas que repercuten en pérdidas de peso y crecimiento. Resultado que difiere a lo reportado por [36], Acero Alarcon [37] al suministrar en la dieta de aves Ross 308; lo que se puede decir que la línea genética utilizada influye sobre el presente parámetro, debido a que obtienen ganancias de pesos superiores. Ya que [23], indica ganancias de pesos menores al suministrar treonina en aves de la línea comercial Arbor Acres Plus; lo que concuerda con lo mencionado por [1], quien habla sobre el impacto potencial de la selección genética que condujo a estudios en cuanto a la digestibilidad relacionada a la composición de la microbiota intestinal, y que respaldado en varios estudios han demostrado que los individuos que se diferencian por su eficiencia alimenticia albergan distintas composiciones de microbiota. Sin embargo algunos estudios compararon líneas de pollos seleccionadas de forma divergente por adiposidad, peso corporal, competencia inmunológica y picoteo de plumas e identificaron diferencias en la composición de la microbiota intestinal de las líneas divergentes. Estos resultados tienden a mostrar que la microbiota cecal del pollo está asociada con el control genético de muchos rasgos de interés [1].

Es importante mencionar que la pared celular de la $S$. Cerevisiae está dispuesta principalmente de complejos de polímeros de $\beta$-glucanos, $\alpha$-mananos, manoproteínas y en menor cantidad quitina. Los mananos y manoproteínas constituyen el 30-40\% de la pared celular y determinan las propiedades de la superficie celular [42]. Mediante las investigaciones en función de estos componentes, se llegó a la conclusión que los glucomananos fosforilados, presentan dos funciones básicas, que se encuentran relacionadas: Influir en la ecología microbiana del intestino y actuar sobre el sistema inmune. En el intestino, actúan seleccionando la presencia de algunas bacterias y eliminando otras, que son nocivas para el ave. Como ejemplo se puede citar, los patógenos 
con fimbrias tipo 1-específicas de manosa, como Escherichia coli y Salmonella, son atraídos por los mananos y se unen inmediatamente con el carbohidrato y no atacan a las células epiteliales del intestino del ave [43]. En el sistema inmune, ayudan a proteger a los pollos de carne de los microorganismos [44].

La adición ligada de las paredes celulares de S. Cerevisiae en diferentes dosis con APC se tradujo a mejores pesos corporales, lo que sugirió un sinergismo entre ambos promotores de crecimiento [41], así lo mencionó [45], quienes hallaron un incremento en el estímulo del sistema retículo endotelial del tracto digestivo, cuando administraron componentes activos de la pared celular y antibióticos al mismo tiempo, y que sin duda favorecen el incremento de la flora benéfica en el tracto intestinal, lo que permite potenciar los beneficios de dichos aditivos, en broilers. Dicho lo cual sería importante, incorporar treonina y Paredes celulares de $S_{\text {. Cerevisiae }}$ en conjunto a la dieta y conocer el sinergismo que podría existir entre ambos promotores de crecimiento.

\subsection{Consumos de alimento de los pollos broiler, $g$}

La variable consumo de alimento a los 14, 28 y 49 días no presentaron diferencias estadísticas $(p>0,05)$. Este hecho estaría determinado, debido que se conservó los niveles de energía metabolizable de los 2 tratamientos versus el testigo, ya que la variación de la densidad energética del alimento es el principal factor que influye sobre el consumo de alimento en aves, por su habilidad para controlar el consumo de energía, siendo mayor cuando la densidad energética es menor, y de manera inversa con la mayor densidad energética [46].

Lo que coincide con los resultados en la etapa inicial, al igual que [47], quienes no registraron diferencias estadísticas. Además, [48] al reemplazar harina de pescado por harina de vísceras en dietas para broiles, no obtuvo diferencias estadísticas para el consumo de alimento al incluir $4 \%$ y $8 \%$ en la etapa de inicio; sin embargo, difiere en la etapa de crecimiento al no presentar diferencias estadísticas para este parámetro. Mientras, que en la etapa de crecimiento los resultados no se encuentran relacionados con los reportados por [49], en pollos de 21-42 días de edad, que indican que el reemplazo de torta de soya por harina de subproductos de aves no presenta diferencias estadísticas en diferentes niveles de inclusiones respecto al tratamiento control, pero se reducen significativamente con niveles de inclusión de $12 \%$ y $15 \%$.

\subsection{Conversiones alimenticias de los pollos broiler a los 28 días}

Para la variable conversión alimenticia de los pollos broiler valorizados a los 28 días, manifestaron diferencias estadísticas altamente significativas $(p<0,01)$, entre los promotores de crecimiento observándose las conversiones menos eficientes para T0 y T1 con 1,44 y 1,43 puntos respectivamente; mientras que T2 resulta ser más eficaz con una media de 1,39 puntos (Tabla 1); posiblemente estos resultados sean producto de la acción de las paredes celulares que aumenta la digestibilidad de nutrientes en el intestino por el aumento del tamaño de las vellosidades [50], ayudan el sistema inmune reduciendo la incidencia de enfermedades respiratorias y otras infecciones 
que se acentúan en períodos de estrés ambiental; debido a que una gran parte de las células inmunológicas en el cuerpo del animal, están localizadas dentro del intestino como parte del tejido linfoide; proporcionando protección inmunológica, tanto específica como no específica, para proteger la superficie del tracto gastrointestinal, reflejándose este sistema inmune en el desarrollo eficiente de los pollos [51]. De manera que incrementa el área de superficie de absorción de los nutrientes y a su vez disminuye la resistencia a antibióticos [52].

Se debe tomar en consideración que la intensa selección llevada a cabo durante las últimas décadas ha supuesto un enorme progreso genético en cuanto a características como la conversión alimenticia y la tasa de crecimiento, por lo que la expresión genética de las mismas está en relación al manejo de la alimentación de los animales con dietas óptimas y fáciles de digerir, compuestas principalmente por piensos que contengan aditivos que no compitan o afecten a la salud humana, pero que a su vez contribuyan al incremento de los parámetros productivos de las aves [1].

\subsection{Peso a la canal de los pollos broiler, $\mathbf{g}$}

En el análisis de la variable peso a la canal de las unidades experimentales, demostraron diferencias estadísticas altamente significativas $(p<0,01)$, entre los promotores de crecimiento en discusión, señalando que el mayor peso a la canal se obtuvo con T2: $2527,05 \mathrm{~g}$; que disminuye a $2450,37 \mathrm{~g}$ con T2; mientras que el menor peso a la canal se registró en las aves To con $2385,40 \mathrm{~g}$, como se indica en la Tabla 2.

Asumiéndose que el bioactivo de la pared celular S. Cerevisiae, ha sido ampliamente utilizada en la alimentación animal como fuente de proteínas y otros nutrientes. Además proporcionan energía, contienen entre $30 \%$ y $70 \%$ de proteína, son ricas en vitaminas del grupo B (B1, B2, B6, ácido pantoténico, niacina, ácido fólico y biotina), minerales, especialmente selenio y fibra que mejoran el rendimiento de las aves gracias a la mayor disponibilidad de nutrientes, los mismos que se desdoblan y se metabolizan con una mejor eficiencia [53].

Referente a la treonina, es indispensable conocer que no es solamente un elemento necesario para la síntesis de proteína, también desempeña un importante papel como aminoácido clave en el metabolismo del intestino y en la respuesta inmunitaria, es por ello la comparación realizada con los componentes bioactivos de S. cerevisiae. Lemme et al. [54] indica que los aminoácidos digestibles ideales estandarizados deben ser revisados debido a datos publicados sobre pérdidas endógenas de aminoácidos en aves. Aunque existen varios métodos para determinar estas pérdidas endógenas, todos ellos indican que las pérdidas de treonina son elevadas en comparación con otros aminoácidos esenciales. Esto puede ser debido a la secreción de mucina, que es el principal constituyente de las pérdidas endógenas y tiene un elevado contenido de treonina [55]. 
Table 2

Efectos de treonina y componentes bioactivos de Saccharomyces cerevisiae sobre el peso y rendimiento a la canal en la producción de aves Cobb 500.

\begin{tabular}{|c|c|c|c|c|c|c|c|c|c|}
\hline \multirow[t]{2}{*}{ Variable } & \multicolumn{6}{|c|}{ Tratamientos } & \multirow[t]{2}{*}{ E.E } & \multirow[t]{2}{*}{ Prob. } & \multirow[b]{2}{*}{ Sig } \\
\hline & TO & & T1 & & T2 & & & & \\
\hline $\begin{array}{l}\text { Peso a la canal, } \\
\text { Kg }\end{array}$ & 2385,40 & c & 2450,37 & $b$ & 2527,05 & a & 14,35 & 0,0001 & $* *$ \\
\hline $\begin{array}{l}\text { Rendimiento a la } \\
\text { canal, \% }\end{array}$ & 79,91 & c & 81,89 & $b$ & 83,85 & a & 0,14 & 0,0001 & $* *$ \\
\hline Mortalidad, No. & 1,00 & & 0,00 & & 0,00 & & & & \\
\hline
\end{tabular}

${ }^{* *}$ Altamente significativo; E.E.: Error estándar.

Prob. > 0,05: No existen diferencias estadísticas; Prob. < 0,05: Existen diferencias estadísticas; Prob. < 0,01: Existen diferencias altamente significativas.

Medias con letras iguales en una misma fila no difieren estadísticamente de acuerdo a la prueba de Tukey.

\subsection{Rendimiento a la canal de los pollos broiler, \%}

Para el rendimiento a la canal en los pollos broiler de la presente investigación, se determinó diferencias estadísticas altamente significativas $(p<0,01)$, registrándose el mayor rendimiento a la canal con T2: 83,85\%; seguido de T1con 81,89\% y finalmente el más bajo rendimiento a la canal fue T0 con 79,91\% Tabla 2, posiblemente se deba a que la biomasa que contiene la levadura natural es usada como complemento alimenticio para animales, dada su alta disponibilidad y bajo costo. Para estos casos, es importante tomar en consideración las dosis que permitan cumplir las funciones de probióticos, con lo que podrían usarse para sustituir total o parcialmente el uso de antibióticos, contribuyendo a mejorar la eficiencia de los sistemas productivos agropecuarios [41, $56,57]$.

Los datos presentados de $2527,05 \mathrm{~g}$, con T1, superan a los registrados al suministrar paredes celulares de S. cerevisiae. Karaoglu y Durdag [58] no reportaron efectos de la suplementación de S. cerevisiae sobre la calidad de la canal; Hernández et al. [56] reportaron un incremento en el rendimiento de la canal por la inclusión de dicho prebiótico, esto puede deberse a que estén asociados a mecanismos fisiológicos de los PCL-Glucanos sobre el tracto gastrointestinal (TGI), como aumento significativo del peso destinado a la canal.

Varios autores mencionan [57] que enriquecer la dieta con S. cerevisiae $(0,5 \%)$, o su pared celular $(0,3 \%)$ o extracto de la misma $(0,3 \%)$, podría aumentar de manera favorable la calidad de la carne de las aves, así como también la terneza y la estabilidad oxidativa de la misma [42], así como también tendría la capacidad de disminuir el nivel de colesterol sérico en broilers [59]; en aves adultas se obtuvieron mejores parámetros productivos las que fueron suministradas extracto de $S$. cerevisiae que las que recibieron la levadura total. Es importante considerar que al usar solo el extracto, se perderían los efectos beneficiosos que aporta la la pared celular, ya que existiría una disminución de la colonización de algunas enterobacterias y favorecimiento del 
cambio morfológico en la mucosa intestinal de los pollos de carne; aun cuando dicha pared no representa un valor nutricional por si misma [60].

Las investigaciones se han enfocado en analizar la calidad de la canal, tomando en consideración las demandas del consumidor, mismas que deben poseer un alto valor nutritivo con bajos niveles de grasa, y que a su vez sean inocuas, lo que se ha logrado agregando probióticos y prebióticos a base de S. cerevisiae [61]; quienes al sustituir dos tercios del núcleo vitamínico-mineral por 0,3\% de dicho probiótico en la fase final de engorde, observaron una mejora en la calidad del peso de la pechuga, muslos, así como también la reducción de manera significativa de grasa abdominal. Mientras que cuando se reemplazó la mitad el núcleo vitamínico mineral por 0,15 y 0,30\% de este probiótico en la fase inicial y engorde, se reportó una disminución no representativa en la grasa abdominal, así como también cierta tendencia de mejora en la deposición de pechuga y muslos de los pollos broilers [61, 62].

Es importante mencionar que los alimentos suplementados con L-treonina tienen un contenido de lisina y aminoácidos azufrados totales significativamente mayor. Además, el contenido de proteína bruta es significativamente menor (0,4 puntos). Los nutricionistas emplean L-treonina como una herramienta para mejorar la calidad del alimento (balance de aminoácidos), pero además también para reducir su costo. Al reducir el contenido de proteína bruta en 0,4 puntos se reduce también proporcionalmente la utilización de ingredientes ricos en proteína; ya que el principal objetivo es reducir el costo de alimentación [41].

\subsection{Mortalidad, No.}

Para la variable mortalidad (No.), en la presente investigación se determinó que no existe una mortalidad alta en los polos evaluados con los diferentes promotores de crecimiento, teniendo el mayor número de mortalidad en el tratamiento testigo con un animal. Resultados que coinciden con [63], donde la mortalidad promedio reportada en su ensayo fue de 1,25\%, debido a que las condiciones ambientales fueron favorables; los datos sugieren que los tratamientos con inclusión de aditivos, presentaron cero mortalidades. Así como también [41] al utilizar diferentes niveles de paredes celulares de $S$. cerevisiae en dos experimentos en broilers, ambas pruebas mantuvieron una respuesta similar en los parámetros de producción y mortalidad a los obtenidos con solamente al aplicar un APC.

\subsection{Análisis económico por efecto de la utilización de treonina y componentes bioactivos de Saccharomyces cerevisiae sobre el peso y rendimiento a la canal en la producción de aves Cobb 500.}

Mediante el análisis económico a través del indicador beneficio/costo de una saca de pollos de engorde (cuadro 10), tomando en consideración los egresos ocasionados y como ingresos la venta los pollos, se estableció la mayor rentabilidad cuando se aplicó la levadura natural, con el cual se obtuvo un beneficio/costo de 1,30, que representa que 
por cada dólar USD invertido, se espera obtener una rentabilidad de 30 centavos USD (30\%), cantidad que se reduce al $26 \%$ (B/C de 1,26), en las aves con dietas adicionadas la treonina, y finalmente al $22 \%$ ( $B / C$ de 1,22 ), con el tratamiento testigo por lo que se considera que mejores índices productivos y económicos se alcanzan al utilizar la levadura natural en pollos de engorde.

\section{Conclusiones}

Los resultados de la presente investigación apuntan a la utilización de componentes bioactivos de $S$. cerevisiae, en dosis de $200 \mathrm{~g} / \mathrm{Tn}$ en la dieta de pollos broilers; principalmente complejos de polímeros de $\beta$-glucanos, $\alpha$-mananos, manoproteínas y en menor cantidad quitina; cuya función de los glucomananos fosforilados, es influir en la ecología microbiana del intestino y actuar sobre el sistema inmune; y a su vez en el intestino, actúan seleccionando la presencia de algunas bacterias y eliminando otras, que son nocivas para el ave. Lo que se respalda con estudios realizados, donde también se recomienda la utilización de dicho prebiótico en las dietas de pollos parrilleros, por ser un producto de origen natural que mejora notoriamente las variables productivas, así como también el rendimiento y la calidad de la canal; y lo más importante siendo amigable con el medio ambiente y de uso seguro para el consumidor (inocuidad alimentaria). Además, se debe tomar en cuenta estudios en los que se destaca la sinergia con otros probióticos y prebióticos incluso llegando a potenciar sus efectos; por lo que sería importante realizar investigaciones de $S$. cerevisiae con treonina en uso conjunto; ya que a partir de la Nutrigenómica se ayuda al semoviente a alcanzar su máximo potencial genético. Lo que se traduce en mejores rendimientos económicos para el productor, en la presente investigación se reportó una rentabilidad del 30\%.

\section{References}

[1] Borey $M$ et al., Broilers divergently selected for digestibility differ for their digestive microbial ecosystems. PloS One. 2020;15(5):e0232418.

[2] Yirga $H$. The use of probiotics in animal nutrition. J. Prob. Health, 2015;3(2):1-10.

[3] Diarra MS, Malouin F. Antibiotics in Canadian poultry productions and anticipated alternatives. Frontiers in microbiology. 2014;5:282.

[4] Qureshi S et al. Histomorphological studies of broiler chicken fed diets supplemented with either raw or enzyme treated dandelion leaves and fenugreek seeds. Vet World. 2016;9(3):269-75.

[5] Pinto S et al. Acción de promotores de crecimiento sobre la mucosa intestinal de pollos parrilleros Action of growth promoters on the intestinal mucosa of broiler chickens.

[6] Muñoz JEM et al. Efecto de la fuente lipídica en la alimentación de pollos para carne en zona de trópico. Revista Investigaciones Agropecuarias. 2020;2(2):31-48.

[7] Patience JF. The influence of dietary energy on feed efficiency in grow-finish swine, in Feed efficiency in swine. Springer; 2012. p. 101-129.

[8] Ewing WN. The living gut. Nottingham University Press; 2009.

[9] Willing BP, Malik G, van Kessel AG. Sustainable swine nutrition. 2012. Nutrition and gut health in swine. p. 197-213.

[10] Rodríguez-Frías JA. Integridad intestinal del pollo de engorde. 2004

[11] Rougière N, Carré B. Comparison of gastrointestinal transit times between chickens from D+ and Dgenetic lines selected for divergent digestion efficiency. Animal. 2010;4(11):1861-72.

[12] Blottière $\mathrm{HM}$ et al. Human intestinal metagenomics: State of the art and future. Curr Opin Microbiol. 2013;16(3):232-9. 
[13] Sommer F, Bäckhed F. The gut microbiota-masters of host development and physiology. Nat Rev Microbiol. 2013;11(4):227-38.

[14] Hanning I, Diaz-Sanchez S. The functionality of the gastrointestinal microbiome in non-human animals. Microbiome. 2015;3:51.

[15] Broom LJ, Kogut $\mathrm{MH}$. The role of the gut microbiome in shaping the immune system of chickens. Vet Immunol Immunopathol. 2018;204:44-51.

[16] Suárez-Machín C, Garrido-Carralero NA, Guevara-Rodríguez CA. Levadura Saccharomyces cerevisiae y la producción de alcohol. Revisión bibliográfica. 2016;50(1):20-28.

[17] Gilka G, Kelly PY. Toxicity comparison of somo animal wastes. Water, Air, Soil pollution. 2015:113-117.

[18] Uscanga BRA, Pacheco JRS, Francios J. Estudio de la variación de la composición de los polisacáridos contenidos en la pared celular de la levadura. Saccharomyces Cerevisiae. e-Gnosis. 2005;3.

[19] Klis FM et al. Dynamics of cell wall structure in Saccharomyces cerevisiae. FEMS microbiology reviews. 2002;26(3):239-256.

[20] Montagne L, Pluske JR, Hampson DJ. A review of interactions between dietary fibre and the intestinal mucosa, and their consequences on digestive health in young non-ruminant animals. Animal feed science and technology. 2003;108(1-4):95-117.

[21] Kim YS, Ho SB. Intestinal goblet cells and mucins in health and disease: Recent insights and progress. Current gastroenterology reports. 2010;12(5):319-330.

[22] Wils-Plotz EL, Dilger RN. Combined dietary effects of supplemental threonine and purified fiber on growth performance and intestinal health of young chicks. Poultry Science. 2013;92(3):726-734.

[23] Chen YP et al., Effects of threonine supplementation on the growth performance, immunity, oxidative status, intestinal integrity, and barrier function of broilers at the early age. Poultry Science. 2017;96(2):405-413.

[24] Law GK et al. Adequate oral threonine is critical for mucin production and gut function in neonatal piglets. American Journal of Physiology-Gastrointestinal and Liver Physiology. 2007;292(5):1293-1301.

[25] Wang W et al. Optimal dietary true ileal digestible threonine for supporting the mucosal barrier in small intestine of weanling pigs. The Journal of nutrition. 2010;140(5):981-986.

[26] Azzam MMM et al. Effect of supplemental L-threonine on mucin 2 gene expression and intestine mucosal immune and digestive enzymes activities of laying hens in environments with high temperature and humidity. Poultry Science. 2011;90(10):2251-2256.

[27] Azzam MMM et al. Influence of L-threonine supplementation on goblet cell numbers, histological structure and antioxidant enzyme activities of laying hens reared in a hot and humid climate. British poultry science. 2012;53(5):640-645.

[28] Ren $\mathrm{M}$ et al. Increased levels of standardized ileal digestible threonine attenuate intestinal damage and immune responses in Escherichia coli K88+ challenged weaned piglets. Animal Feed Science and Technology. 2014;195:67-75.

[29] Trevisi P et al. Effect of added dietary threonine on growth performance, health, immunity and gastrointestinal function of weaning pigs with differing genetic susceptibility to $E$ scherichia coli infection and challenged with E. coli K88ac. Journal of animal physiology and animal nutrition. 2015;99(3):511520.

[30] Parmentier HK et al., Serum haemolytic complement activities in 11 different MHC (B) typed chicken lines. Veterinary Immunology and Immunopathology. 2004;100(1-2):25-32.

[31] Wils-Plotz EL, Jenkins MC, Dilger RN. Modulation of the intestinal environment, innate immune response, and barrier function by dietary threonine and purified fiber during a coccidiosis challenge in broiler chicks. Poultry Science. 2013;92(3):735-745.

[32] Macavilca Y, Vergara V, Valverde N. Efecto de un concentrado proteico a base de subproductos de camal avícola sobre el comportamiento productivo de pollos de carne. Agroindustrial Science. 2020;10(2):129-135.

[33] Upendra HA, Yathiraj S. Effect of supplementing probiotics and Mannan Oligosaccharide on body weight, feed conversion ratio and livability in broiler chicks. Indian veterinary journal. 2003;80(10):10751077.

[34] Waldroup PW, Fritts CA, Yan F. Utilization of Bio-Mos ${ }^{\circledR}$ mannan oligosaccharide and Bioplex ${ }^{\circledR}$ copper in broiler diets. International Journal of Poultry Science. 2003;2(1):44-52.

[35] Santin, E., et al., Evaluation of the efficacy of Saccharomyces cerevisiae cell wall to ameliorate the toxic effects of aflatoxin in broilers. International Journal of Poultry Science, 2003. 2(5): p. 341-344.

[36] Saadatmand N, Toghyani M, Gheisari A. Effects of dietary fiber and threonine on performance, intestinal morphology and immune responses in broiler chickens. Animal Nutrition. 2019;5(3):248-255.

[37] Alarcon AF. Evaluacion del efecto de tres niveles de treonina en ganancia de peso en pollos parrilleros Linea Ross 308 en la colonia Florida provincia Caranavi La Paz. 2016. 
[38] Dildey $D$ et al. Effect of mannan oligosaccharide supplementation on performance and health of Holstein calves. Journal of Dairy Science. 1997;80(suppl 1);188-189.

[39] Reyes-Sánchez $\mathrm{N}$ et al. Rendimiento de la canal y morfometría del tracto gastrointestinal de broilers suplementados con pared celular de levadura. La Calera. 2014;14(22):33-37.

[40] López RM. Las paredes celulares de levadura de Saccharomyces cerevisiae: Un aditivo natural capaz de mejorar la productvidad y salud del pollo de engorde. 2008.

[41] Menocal JA et al. Efecto de paredes celulares (Saccharomyces cerevisiae) en el alimento de pollo de engorda sobre los parámetros productivos. Revista Mexicana de Ciencias Pecuarias. 2005;43(2):155162.

[42] Zhang AW et al., Effects of yeast (Saccharomyces cerevisiae) cell components on growth performance, meat quality, and ileal mucosa development of broiler chicks. Poultry science. 2005;84(7):1015-1021.

[43] Pérez-Sotelo LS et al. In vitro evaluation of the binding capacity of Saccharomyces cerevisiae Sc47 to adhere to the wall of Salmonella spp. Rev Latinoam Microbiol. 2005;47(3-4):70-75.

[44] Khati BM et al. Effect of low protein level supplemented with or without yeast (Saccharomyces cerevisiae) on hematological and immunological profile of broiler quails. Royal Veterinary Journal of India. 2007;3(2):131-136

[45] Lahnborg G, Hedström KG, Nord CE. The effect of glucan-a host resistance activator-and ampicillin on experimental intraabdominal sepsis. Journal of the Reticuloendothelial Society. 1982;32(5):347-353.

[46] Saleh EA et al. Effects of dietary nutrient density on performance and carcass quality of male broilers grown for further processing. International Journal of Poultry Science. 2004.

[47] Jafari $\mathrm{M}$ et al. Evaluation of protein and energy quality of poultry by-product meal using poultry assays. African Journal of Agricultural Research. 2011;6(6):1407-1412.

[48] Hossain MH, Ahammad MU, Howlider MAR. Replacement of fish meal by broiler offal in broiler diet. International Journal of Poultry Science. 2003;2(2):159-163.

[49] Hassanabadi A, Amanloo H, Zamanian M. Effects of substitution of soybean meal with poultry by-product meal on broiler chickens performance. Journal of Animal and Veterinary Advances. 2008;7(3):303-307.

[50] Yang Y, lji PA, Choct M. Effects of different dietary levels of mannanoligosaccharide on growth performance and gut development of broiler chickens. Asian-Australasian Journal of Animal Sciences. 2007;20(7):1084-1091.

[51] Liao SF, Nyachoti M. Using probiotics to improve swine gut health and nutrient utilization. Animal Nutrition. 2017;3(4):331-343.

[52] Cruickshank G. Gut microflora the key healthy broiler growing. Poultry World. 2002;156(7):14.

[53] Spring $P$ et al. The effects of dietary mannaoligosaccharides on cecal parameters and the concentrations of enteric bacteria in the ceca of salmonella-challenged broiler chicks. Poultry science. 2000;79(2):205-211.

[54] Lemme A, Ravindran V, Bryden WL. Ileal digestibility of amino acids in feed ingredients for broilers. World's Poultry Science Journal. 2004;60(4):423-438.

[55] Donato DCZ et al. Manipulation of dietary methionine+ cysteine and threonine in broilers significantly decreases environmental nitrogen excretion. Animal. 2016;10(6):903-910.

[56] Hernández NL, Téllez GA, Nieto CJA. Evaluación de tres levaduras provenientes de ecosistemas colombianos en la alimentación de pollos de engorde. Ciencia \& Tecnología Agropecuaria. 2009;10(1):102-114.

[57] Peralta MF, Miazzo RD, Nilson A. Levadura de cerveza (Saccharomyces cerevisiae) en la alimentación de pollos de carne. REDVET. Revista electrónica de Veterinaria. 2008;9(10):1-11.

[58] Karaoglu M, Durdag H. The influence of dietary probiotic (Saccharomyces cerevisiae) supplementation and different slaughter age on the performance, slaughter and carcass properties of broilers. International Journal of Poultry Science. 2005;4(5):309-316.

[59] Lee BD et al. Effects of dietary yeast (Saccharomyces cerevisiae) components on growth performance and cholesterol metabolism in broiler chickens. Kor. J. Poult. Sci. 2005;32:49-54.

[60] Perdomo MC, Vargas RE, Campos J. Valor nutritivo de la levadura de cervecería (Saccharomyces cerevisiae) y de sus derivados, extracto y pared celular, en la alimentación aviar. Archivos Latinoamericanos de Producción Animal. 2004;12(5):89-95

[61] Miazzo RD, Peralta MF. Calidad de la canal de pollos parrilleros que recibieron Levadura de Cerveza (S. cerevisiae) en sustitución del núcleo vitamínico-mineral. REDVET. Revista Electrónica de Veterinaria. 2006;7(11):1-7.

[62] Miazzo RD et al. Calidad de la canal de broilers que recibieron levadura de cerveza (S. cerevisiae) en las etapas de iniciación y terminación. XX Cong. Latinoam. De Avicultura: NU; 2007.

[63] Castro M. Comportamiento productivo en pollos de engorde alimentados con niveles crecientes de alcachofa (Cynara scolymus). Revista Colombiana de Ciencia Animal. 2016;9(1). 